\title{
Criminologie
}

\section{Trajectoires criminelles et récidive des délinquants sexuels adultes : l'hypothèse " statique " revue et corrigée}

\section{Patrick Lussier}

Volume 43, numéro 2, automne 2010

Les 50 ans de l’École de criminologie : aperçu de la recherche d'ici et d'ailleurs

URI : https://id.erudit.org/iderudit/1001778ar

DOI : https://doi.org/10.7202/1001778ar

Aller au sommaire du numéro

Éditeur(s)

Les Presses de l’Université de Montréal

ISSN

0316-0041 (imprimé)

1492-1367 (numérique)

Découvrir la revue

Citer cet article

Lussier, P. (2010). Trajectoires criminelles et récidive des délinquants sexuels adultes : l'hypothèse « statique » revue et corrigée. Criminologie, 43(2), 269-302. https://doi.org/10.7202/1001778ar
Résumé de l'article

De façon générale, l'évaluation du risque repose sur des outils actuariels qui incluent des facteurs statiques ou historiques tels les antécédents criminels. La présente étude réexamine la relation entre les antécédents criminels et la récidive violente/sexuelle en tenant compte de l'aspect dynamique de la carrière criminelle. L'étude pose la question suivante : est-ce que les trajectoires criminelles informent sur les risques de récidive ? L'étude actuelle est basée sur une quasi-population de délinquants sexuels adultes incarcérés dans la province de Québec entre 1994 et 2000. Des analyses de classes latentes ont été réalisées afin d'identifier les trajectoires criminelles violentes/sexuelles. Les analyses de survie indiquent que les trajectoires criminelles informent sur les risques de récidive violente/sexuelle tout en montrant des limites importantes de la prédiction actuarielle. 


\title{
Trajectoires criminelles et récidive des délinquants sexuels adultes: l'hypothèse "statique » revue et corrigée
}

\author{
Patrick Lussier \\ Centre for Research on Sexual Violence \\ School of Criminology, Simon Fraser University \\ plussier@sfu.ca
}

RÉSUMÉ - De façon générale, l'évaluation du risque repose sur des outils actuariels qui incluent des facteurs statiques ou historiques tels les antécédents criminels. La présente étude réexamine la relation entre les antécédents criminels et la récidive violente/ sexuelle en tenant compte de l'aspect dynamique de la carrière criminelle. L'étude pose la question suivante: est-ce que les trajectoires criminelles informent sur les risques de récidive? L'étude actuelle est basée sur une quasi-population de délinquants sexuels adultes incarcérés dans la province de Québec entre 1994 et 2000. Des analyses de classes latentes ont été réalisées afin d'identifier les trajectoires criminelles violentes/ sexuelles. Les analyses de survie indiquent que les trajectoires criminelles informent sur les risques de récidive violente/sexuelle tout en montrant des limites importantes de la prédiction actuarielle.

MOTS-CLÉS - Délinquants sexuels, trajectoires criminelles, prédiction actuarielle, récidive, évaluation du risque, dangerosité, âge, antécédents judiciaires.

\section{Introduction}

$\mathrm{Au}$ Canada, depuis plus de quinze ans déjà, plusieurs mesures pénales ont été implantées afin de gérer le risque que représentent les délinquants sexuels pour la collectivité. La loi relative aux délinquants dangereux, une ordonnance de surveillance à long terme en communauté, et la section 810 du Code criminel (i.e., ordre de ne pas troubler la paix) ont été mises en œuvre afin: a) de neutraliser les délinquants sexuels en prison pour de plus longues périodes; b) d'augmenter les 
mécanismes de surveillance pour ceux qui réintègrent la collectivité. Sauf exception (par exemple, le registre de délinquants sexuels), au Canada, contrairement aux États-Unis, ces mesures ne ciblent pas tous les délinquants sexuels, mais visent plutôt les individus qui représentent un risque élevé de récidive (Lussier et al., 2010). Ces mesures pénales sont clairement destinées à neutraliser les délinquants à risque élevé tout en augmentant la protection de la collectivité (Petrunik, 2002). Le «modèle canadien de protection communautaire» représente en fait une approche jugée par certains plus prudente comparativement à celle en place aux États-Unis (Petrunik, 2003). D’ailleurs, les criminologues étatsuniens ont sévèrement critiqué les nouvelles mesures pénales étatsuniennes sur plusieurs fronts: a) l'absence d'un effet significatif sur la récidive; b) les conséquences négatives sur la capacité des délinquants à réintégrer avec succès la collectivité après avoir purgé leur peine de prison (Simon, 1998; Lafond, 2005 ; Tewsbury, 2005 ; Levenson et al., 2007). Aux États-Unis, en outre, les coûts associés à l'utilisation accrue de la prison ainsi que des peines plus longues et fixes ont mené à une baisse des possibilités de traitement et de réadaptation (Travis et Petersilia, 2001). Au Canada, tandis que les efforts de réhabilitation restent une partie intégrante de l'objectif de justice pénale, le traitement a été mis en arrière-plan à l'égard des objectifs de gestion du risque, et ce, en dépit de preuves empiriques qui suggèrent un effet positif des programmes de traitement des délinquants sexuels (par exemple Hanson et al., 2002; Lösel et Schmucker, 2005; Mackenzie, 2006).

Cette gestion du risque a été élaborée à partir de l'hypothèse qu'il y a une certaine stabilité de l'activité criminelle et du risque de récidive des délinquants sexuels. Cette hypothèse est généralement soutenue par des études empiriques réalisées auprès d'échantillons hautement sélectifs de délinquants sexuels (Prentky et al., 1997; Doren, 1999). En conséquence, au fil des années, des termes tels que «dangerosité», «troubles mentaux» et «spécialisation criminelle» ont été souvent associés aux délinquants sexuels (Simon, 2000). Ces affirmations tracent un profil du risque représenté par les délinquants sexuels comme statique ou immuable à travers le temps. En d'autres termes, selon cette perspective, le risque de récidive chez les délinquants sexuels demeure stable en dépit de facteurs tels que l'âge et le vieillissement (Lussier et Healey, 2009). Selon plusieurs chercheurs, cette stabilité du risque de récidive peut être évaluée par des instruments de prédiction dits «actuariels» qui incluent des prédicteurs «statiques» ou «historiques». 
Lutilisation de ces outils fait partie des pratiques courantes au sein des différents milieux correctionnels canadiens et étatsuniens. La présente étude s'attarde à un des prédicteurs statiques les plus couramment utilisés par les évaluateurs du risque et qui occupent une place prépondérante parmi les instruments de prédiction: les antécédents criminels. Si la relation entre le comportement passé et futur semble bien établie, elle demeure imparfaite malgré la présence de corrélations significatives et influencée par le passage du temps. Le but de cette étude empirique est donc de clarifier ces points chez un groupe de délinquants sexuels adultes. Plus spécifiquement, l'étude aborde la relation entre le comportement criminel passé et futur en ayant recours à une approche centrée sur la carrière criminelle des délinquants sexuels. Ce faisant, l'étude aborde le comportement criminel des délinquants sexuels dans une perspective dite dynamique centrée sur l'individu et les patrons de comportements criminels à travers le temps, créant ainsi une coupure avec les études précédentes où l'approche actuarielle, statique et centrée sur les variables prédomine.

\section{L'évaluation et la prédiction actuarielle du risque de récidive}

«The new penology» a marqué un changement graduel des pratiques pénales en reconnaissant l'hétérogénéité des délinquants en fonction de leur risque de récidive (Feeley et Simon, 1992). Depuis les premiers balbutiements de l'évaluation du risque (notamment, Sarbin, 1943; Meehl, 1954; Nuffield, 1982; Quinsey et al., 1995), différents outils ont émergé dans les années 1990 qui aident à classer les délinquants sexuels selon leur risque de récidive: RRASOR (Hanson, 1997), SORAG (Quinsey et al., 1998), MnSOST-R (Epperson et al., 1998), Static-99 (Hanson et Thornton, 2000). Ces outils actuariels ont été mis au point afin de classer les délinquants sexuels selon le niveau de risque de récidive (c'est-à-dire, faible, moyen, haut risque). Les outils de prédiction actuariels sont basés principalement sur une série de prédicteurs statiques et dynamiques associés empiriquement à la récidive sexuelle. Les facteurs dynamiques sont ceux qui renseignent sur le risque de récidive, mais également sur les changements associés au risque de récidive (Proulx et al., 2000). Ces facteurs sont souvent décrits comme ceux qui devraient faire l'objet d'un traitement ou d'une intervention, notamment des distorsions cognitives, une régulation personnelle inadéquate, un manque d'empathie, des préférences sexuelles déviantes, 
des déficits sur le plan de l'intimité, etc. (Hanson et Harris, 2001; Ward et Beech, 2006; Lussier et Cortoni, 2008).

Si les facteurs dynamiques sont généralement utilisés pour des fins de traitement, l'évaluation du risque tend à reposer surtout sur des prédicteurs statiques. Les prédicteurs statiques sont ceux qui caractérisent l'histoire de l'individu et qui ne peuvent pas être modifiés par une intervention ou par un programme de traitement (Proulx et al., 2000). Par exemple, le Static-99 est l'un des instruments largement utilisés afin d'évaluer le risque de récidive statique des délinquants sexuels adultes (Doren, 2004). Cet outil est basé sur les facteurs de risque statiques liés à l'histoire criminelle de l'individu, tels que la présence d'un antécédent de crime avec violence, le nombre total de condamnations antérieures pour un crime sexuel, et le nombre total de condamnations antérieures. Des items similaires sont inclus dans les autres outils actuariels de prédiction, notamment la présence d'une révocation de la libération conditionnelle (SORAG; MnSOST-R) ainsi que la présence d'antécédents de crimes contre les biens (SORAG). Le Static-99 est fondé sur l'hypothèse de linéarité entre le nombre de prédicteurs "présents» et la probabilité de récidive. Ainsi, plus le nombre de facteurs de risque statiques est élevé, et ceux-ci s'accumulent au fil des condamnations, plus le risque de récidive, tel qu'évalué par l'outil, augmente. Par conséquent, les individus ayant de longs dossiers criminels sont susceptibles d'être classés comme étant à haut risque de récidive. Il n'est pas surprenant de constater que les récidivistes dans la quarantaine sont souvent ciblés par les politiques qui visent les agresseurs sexuels à haut risque de récidive (Lussier et al., 2009). Cela soulève la question suivante: est-ce que les délinquants sexuels représentent le même risque de récidive tout au long de leur vie?

\section{Le vieillissement comme facteur modérateur du risque de récidive}

Au cours des dernières années, plusieurs études se sont attardées au rôle de l'âge sur les risques de récidive des délinquants sexuels (Hanson, 2002, 2006; Barbaree et al., 2003 ; Doren, 2006; Fazel et al., 2006; Thornton, 2006; Barbaree et al., 2007; Harris et Rice, 2007; Prentky et Lee, 2007; Barbaree et Blanchard, 2008; Lussier et Healey, 2009). Ces études empiriques ont montré une relation modeste entre l'âge et la récidive sexuelle. Une méta-analyse sur les prédicteurs de la récidive sexuelle a montré une relation inverse d'environ 0,10 entre l'âge du 
délinquant et la récidive générale et la récidive sexuelle (Hanson et Bussière, 1998). Par ailleurs, les études descriptives ont montré que les délinquants sexuels dans la vingtaine représentent le groupe le plus susceptible de récidiver alors que ceux dans la cinquantaine montrent des taux de récidive beaucoup plus faibles (Barbaree et al., 2003; Wollert, 2006). À cet égard, Hanson (2006) a montré que le taux de récidive sexuelle des délinquants sexuels plus âgés est inférieur à $2 \%$ sur une période de cinq ans suivant la libération (voir également Thornton, 2006; Lussier et Healey, 2009). En lien avec ces observations, les analyses de survie suggèrent que le risque de récidive des délinquants sexuels adultes diminue de 2 à $4 \%$ par année (Barbaree et al., 2003; Hanson, 2006; Lussier et Healey, 2009; Thornton, 2006). Ces résultats ont en outre conduit certains chercheurs à faire valoir l'importance d'établir des taux de base de la récidive sexuelle en fonction de l'âge du délinquant (Wollert, 2006). D'autres ont contesté cette interprétation, affirmant que la relation négative observée dans les études précédentes ne serait que le résultat de l'agrégation des taux de récidive de jeunes délinquants sexuels et de ceux plus âgés, plutôt que d'une baisse constante des risques de récidive à travers le temps (Doren, 2006; Thornton, 2006). Si le facteur de vieillissement doit être pris en compte afin d'évaluer le risque de récidive, et ce, même chez les délinquants chroniques, alors la relation entre le comportement passé et le comportement futur doit être forcément réexaminée par la communauté scientifique. Une des raisons qui expliquent la difficulté de bien saisir l'association entre le passé criminel, le vieillissement et le risque de récidive est que l'approche actuarielle actuelle est centrée sur les variables.

\section{Approche actuarielle centrée sur les «variables»}

La recherche empirique portant sur l'évaluation et la prédiction du risque est typiquement centrée sur les variables. Les variables, ou facteurs de risque, représentent l'unité d'analyse principale des chercheurs intéressés à prédire le risque de récidive. Selon cette approche, le focus est mis sur l'identification des facteurs associés à la récidive sexuelle. Cette approche tend à mettre l'accent sur les différences individuelles stables qui informent sur les probabilités de récidive à long terme. L'approche centrée sur les variables a recours à une série de "prédicteurs» dont l'information est agrégée en un score total qui représente les probabilités de récidive. Les variables qui sont plus fortement 
associées à la récidive prennent plus d'importance dans la comptabilisation du score de risque. Selon cette approche, la combinaison ou l'agencement particulier de facteurs n'est pas important; c'est plutôt le nombre de facteurs de risque présents. Dans l'approche actuarielle centrée sur les variables, le risque de récidive est perçu comme étant associé de façon linéaire à la récidive. Les délinquants peuvent donc être classés selon un continuum de scores qui permet de les catégoriser selon le niveau de risque qu'ils représentent. Selon l'approche actuarielle, le score actuariel permet de mesurer un trait sous-jacent qui informe sur les risques de persistance du comportement criminel sexuel (Roberts et al., 2002; Barbaree et al., 2009). Bref, un score plus élevé indique la présence de ce trait qui prédispose les individus à continuer leurs activités criminelles. L'approche actuarielle centrée sur les variables est donc basée sur: a) l'hypothèse selon laquelle les risques de récidive sont hétérogènes d'un individu à l'autre; b) cette hétérogénéité peut être mesurée par l'accumulation (ou l'absence) de facteurs associés à la récidive; c) cette accumulation est associée de façon linéaire aux risques de récidive; d) les différences individuelles associées à la récidive sont relativement stables à travers le temps. Dans cette optique, les antécédents judiciaires et l'âge du délinquant constituent deux facteurs de risque distincts qui informent sur les risques de récidive. Par exemple, selon le Static-99 (Hanson et Thornton, 2000), les individus ayant un antécédent judiciaire pour un crime sexuel, de même que ceux âgés de moins de 25 ans au moment de l'évaluation, sont plus à risque de récidive. L'instrument ne permet pas d'ajuster à la baisse les risques de récidive des individus plus âgés ni le passage du temps. Autrement dit, l'instrument ne tient pas en compte le fait que plusieurs années ont pu s'écouler depuis les condamnations antérieures. Ce faisant, ce type d'instrument ne tient pas en compte l'aspect dynamique de l'activité criminelle - c'est-à-dire si l'activité criminelle de l'individu connaît un ralentissement significatif ou une accélération importante. Cette approche contraste avec celle centrée sur la personne, dont l'aspect dynamique du changement du comportement est pris en compte dans une perspective longitudinale.

\section{Approche centrée sur la personne}

Depuis quelques années déjà, certains chercheurs critiquent ouvertement les instruments de prédiction actuariels du fait qu'ils ne permettent 
pas d'évaluer le risque d'une façon plus individualisée et non linéaire (Silver et al., 2000; Côté, 2001 ; Monahan et al., 2005). Plus spécifiquement, il y a différents types de délinquants sexuels et de profils de risque de récidive qui ne peuvent pas être bien évalués par un seul instrument basé sur une approche centrée sur les variables ou sur les facteurs de risque. Cette limite a été soulevée dans un contexte assez différent, mais dont les implications s'adaptent au contexte de l'évaluation du risque. En effet, les chercheurs en psychologie et en criminologie développementale ont démontré que les changements dynamiques de la personne ne sont pas bien pris en compte dans une perspective centrée sur les variables. L'approche centrée sur les variables est basée sur des données agrégées et des scores moyens qui ne permettent pas de bien représenter les différents patrons individualisés de développement (Von Eye et Bergman, 2003; Von Eye et Bogat, 2005). Par conséquent, les conclusions tirées des études portant sur une approche centrée sur les variables peuvent ne pas s'appliquer à une majorité d'individus puisque les résultats reflètent des tendances moyennes. Un parallèle peut être établi entre, d'un côté, les études portant sur les prédicteurs de la récidive qui présentent des tendances générales sur les facteurs associés à la récidive, et de l'autre côté, un psychologue qui doit se pencher sur un cas précis afin d'en évaluer le risque. Lapproche développementale propose en revanche un paradigme distinct qui recadre le focus sur la personne et les différents patrons de développement à travers le temps (Bergman et Magnusson, 1997). Ainsi, afin de mieux rendre compte de l'hétérogénéité des patrons de développement, l'approche centrée sur la personne a été proposée pour désagréger l'information en identifiant des patrons de développement, tout en considérant que certains patrons sont plus fréquents que d'autres (Bergman, 2002). En ce sens, le développement peut être conceptualisé comme un processus caractérisé par un état qui peut changer à travers le temps (Bergman et Magnusson, 1997), attribuable à deux aspects sous-jacents du développement, soit le temps et le changement. Par conséquent, les mesures répétées sont importantes afin de repérer la continuité et le changement à travers le temps. Pour ce faire, la modélisation du développement du comportement doit prendre en compte la constante (c.-à-d. le niveau initial du comportement) et la pente (c.-à-d. la tendance à travers le temps). Ainsi, l'approche centrée sur la personne passe par la modélisation de mesures répétées qui rend compte de la diversité des trajectoires de développement dans une perspective longitudinale. 


\section{Approche dynamique de l'activité criminelle}

L'approche développementale centrée sur la personne, qui n'est pas sans rappeler l'étude de la carrière criminelle (Blumstein et al., 1986; Farrington, 2005), a été adaptée en criminologie pour étudier le développement du comportement délinquant et criminel (Le Blanc et Fréchette, 1989). Laspect dynamique du comportement criminel passe par une description des différents paramètres de son développement: l'activation, l'aggravation et le désistement. On comprend mieux l'importance de mesures répétées du comportement criminel à travers le temps afin d'en décrire son développement et à travers ces différentes phases. L'approche centrée sur la personne suggère que différents patrons d'activation, d'aggravation et de désistement caractérisent le développement du comportement criminel. Autrement dit, les tenants de l'approche développementale suggèrent la présence d'une diversité de trajectoires criminelles. À cet égard, plusieurs modèles de classification ont été élaborés afin de rendre compte et d'expliquer cette diversité (Moffitt, 1993; Le Blanc, 2005; Thornberry et Krohn, 2005). Ces modèles de classification se distinguent à plusieurs égards. Certains chercheurs stipulent que ces trajectoires sont qualitativement différentes et influencées par des combinaisons de facteurs distincts (notamment Moffitt, 1993). D'autres suggèrent que les mêmes facteurs sont en cause, mais ceux-ci varient en intensité d'une trajectoire à l'autre (notamment Le Blanc, 2005). Ces modèles de classification ont été élaborés principalement afin de rendre compte du développement du comportement antisocial durant l'enfance et de la délinquance à l'adolescence. À cet égard, les études portant sur l'analyse des trajectoires de comportements criminels à l'âge adulte sont peu nombreuses. De plus, ces modèles de classification ont été élaborés à partir d'une population générale de délinquants, limitant ainsi les conclusions pouvant être tirées concernant des sous-populations spécifiques comme les délinquants sexuels adultes. L'approche dynamique centrée sur la personne contraste donc avec l'approche actuarielle qui est transversale et axée sur l'identification de facteurs de risque associés de façon linéaire au risque de récidive. Bref, si l'approche actuarielle centrée sur les variables ne s'intéresse qu'à la présence et à la nature des antécédents judiciaires de l'individu au moment de l'évaluation du risque du fait qu'ils constituent des facteurs de risque statique, l'approche dynamique centrée sur la personne s'intéresse à la trajectoire criminelle de l'individu à travers le temps, principalement à son activation, à son aggravation et au désistement. 


\section{Buts de l'étude}

La prédiction actuarielle repose principalement sur la présence d'antécédents criminels afin de déterminer les risques de récidive et, de ce fait, la dangerosité du délinquant. Ce faisant, la prédiction actuarielle repose sur une conception statique du délinquant sexuel dans la mesure où l'âge du délinquant au moment de commettre ses crimes antérieurs et le temps passé depuis ne sont pas tenus en compte par les évaluateurs du risque. Le but de la présente étude est d'aborder la prédiction du risque dans une perspective centrée sur la personne. En outre, l'étude vise à établir un pont entre la littérature scientifique développementale et celle portant sur l'évaluation du risque chez des délinquants sexuels adultes. Pour ce faire, la relation entre les antécédents judiciaires, l'âge, le passage du temps et la récidive a été abordée dans une perspective longitudinale. Plusieurs études en criminologie ont été réalisées afin de désagréger l'information sur l'activité criminelle et d'en examiner les différents patrons de trajectoire criminelle (pour une synthèse de ces travaux, voir Le Blanc, 2005 ; Piquero, 2008). Il est difficile de tirer des conclusions fermes quant aux résultats de ces études, considérant que différents paramètres méthodologiques varient d'une étude à l'autre, notamment la nature de l'échantillon (population générale, échantillon à risque, échantillon de délinquants), le comportement analysé (crimes contre les biens, crimes violents, comportements agressifs), la source d'information utilisée (données policières, données auto-révélées), la période de vie (enfance, adolescence, âge adulte) et la durée du suivi (5, 10, 15 ans), etc. Plus important encore, seulement une étude a été réalisée auprès d'un échantillon de délinquants sexuels adultes (Lussier et al., 2010). Toutefois, elle a été réalisée afin d'identifier les trajectoires criminelles générales. Aucune étude à ce jour n’a examiné les trajectoires de comportements criminels violents et sexuels. De plus, aucune étude à ce jour n'a établi de rapprochement entre les trajectoires criminelles et les risques de récidive après la libération.

\section{Méthodologie}

\section{Échantillon}

Dans le cadre d'un projet de recherche sur la récidive, tous les individus admis de façon consécutive dans un pénitencier fédéral pour un crime sexuel entre avril 1994 et juin 2000 ont été recrutés pour une étude. 
Tous ces individus ont été condamnés à une peine de prison d'au moins deux ans à être purgée dans un pénitencier fédéral dans la province de Québec, au Canada. Au total, $93 \%$ des individus sollicités $(n=553)$ ont accepté de participer à cette étude. La présente étude est basée sur les 393 premiers cas recrutés puisque des données détaillées sur la carrière criminelle n'étaient disponibles dans les dossiers que pour les 393 premiers cas recrutés. Parmi ces personnes, seulement les délinquants âgés d'au moins 30 ans au moment de leur libération $(\mathrm{n}=310)$ ont été retenus pour les fins de cette étude. La décision de ne conserver que les délinquants âgés de plus de 30 ans s'explique du fait que le focus de cette étude est mis: a) sur les délinquants sexuels plus âgés; b) sur les groupes d'âge moins susceptibles de récidiver à leur sortie de prison. De plus, le cadre analytique de cette étude nécessitait une période d'observation suffisamment longue en ce qui concerne les antécédents judiciaires (entre 18 et 30 ans) afin de permettre d'évaluer et de comparer les processus statique et dynamique ${ }^{1}$. Les informations descriptives de l'échantillon sont présentées dans le tableau 1.

TABLEAU 1

Information descriptive de l'échantillon de délinquants sexuels

\begin{tabular}{|r|c|c|}
\hline \multicolumn{1}{|l|}{ Caractéristiques } & $\begin{array}{c}\text { Moyenne, écart-type, } \\
\text { étendue }\end{array}$ & Pourcentage \\
\hline Âge à la sortie de prison & $44,94(10,50)(30-77)$ & - \\
\hline $30-39$ & & 36,0 \\
\hline $40-49$ & & 33,4 \\
\hline $50-59$ & & 19,9 \\
\hline 60 et plus & & 10,6 \\
\hline Éducation (secondaire non complété) & - & 62,7 \\
\hline Origine ethnique & & 91,1 \\
\hline Caucasienne & - & 4,2 \\
\hline Noire & - & 2,6 \\
\hline Autochtone & - & 2,1 \\
\hline Autre & - & - \\
\hline
\end{tabular}

1. Au moment de l'étude, tous les participants étaient incarcérés au Centre régional de réception de Sainte-Anne-des-Plaines, un établissement à sécurité maximale géré par le Service correctionnel du Canada. Durant cette période, la durée moyenne de séjour dans cette institution était d'environ six semaines, permettant ainsi de compléter l'évaluation de l'individu afin de l'assigner à un pénitencier adapté à son niveau de risque et à ses besoins de traitement. 
TABLEAU 1 (suite)

\begin{tabular}{|c|c|c|}
\hline Caractéristiques & $\begin{array}{c}\text { Moyenne, écart-type, } \\
\text { étendue }\end{array}$ & Pourcentage \\
\hline Durée de la période de suivi (mois) & $61,52(20,93)(1-120)$ & - \\
\hline $\begin{array}{l}\text { Nombre de charges pour crime violent/ } \\
\text { sexuel (entre } 18 \text { et } 29 \text { ans) }\end{array}$ & $1,05(2,04)(0-16)$ & - \\
\hline \multicolumn{3}{|l|}{ Antécédent pour un crime violent (présence) } \\
\hline $18-20$ & - & 9,0 \\
\hline $27-29$ & - & 8,4 \\
\hline $18-29$ & - & 29,0 \\
\hline \multicolumn{3}{|l|}{ Antécédent pour un crime sexuel (présence) } \\
\hline $18-20$ & - & 3,5 \\
\hline $27-29$ & - & 5,8 \\
\hline $18-29$ & - & 15,1 \\
\hline \multicolumn{3}{|l|}{$\begin{array}{l}\text { Antécédent pour un crime violent/sexuel } \\
\text { (présence) }\end{array}$} \\
\hline $18-20$ & - & 11,3 \\
\hline $27-29$ & - & 13,2 \\
\hline $18-29$ & - & 35,2 \\
\hline \multicolumn{3}{|l|}{ Récidive (présence) } \\
\hline Sexuelle & - & 5,1 \\
\hline Violente/sexuelle & - & 13,3 \\
\hline
\end{tabular}

\section{Procédures}

Cette étude portant sur la prédiction de la récidive a été approuvée par le comité d'éthique de l'Université de Montréal. Tous les individus qui y ont participé ont été rencontrés individuellement par un assistant de recherche (étudiant aux études supérieures en criminologie) formé par un psychologue. La participation à cette étude a été strictement volontaire, et les participants ont signé un formulaire de consentement indiquant que les informations recueillies seraient utilisées uniquement à des fins de recherche. Les participants ont signé un formulaire de consentement permettant l'accès à leurs dossiers correctionnels. Les dossiers correctionnels ont été utilisés pour colliger les données sur les antécédents judiciaires. Les données sur les antécédents judiciaires sont toutes basées sur les informations fournies par la Gendarmerie royale du Canada. Les données sur la récidive ont été colligées en juin 2004. 


\section{Mesures}

Antécédents judiciaires. Le nombre de charges pour un crime violent ou sexuel a été comptabilisé dans une optique longitudinale. Ainsi, toutes les charges ayant été reçues entre l'âge de 18 et 29 ans ont été colligées, et ce, en tenant compte de quatre périodes distinctes en vertu de l'âge du délinquant au moment de recevoir ces charges: a) entre 18 et 20 ans; b) entre 21 et 23 ans; c) entre 24 et 26 ans; d) entre 27 et 29 ans. Cette période d'observation d'une durée d'un peu plus de 10 ans est d'autant plus pertinente à l'examen des trajectoires criminelles sexuelles et violentes si l'on considère que les études sur la récidive montrent que cette période est celle où les taux de récidive sont les plus élevés (Wollert, 2006). Considérant que cet échantillon ne compte que peu d'antécédents judiciaires pour un crime violent/sexuel, la décision a été prise de ne tenir compte que de la présence d'au moins une charge pour un crime violent et/ou sexuel (et non du nombre de charges). La présence d'antécédents judiciaires pour un crime violent et/ou sexuel pour chacune des quatre périodes a été codée en deux catégories $(0=$ absence de charge; 1 = présence d'au moins une charge). Les données descriptives sont présentées dans le tableau 1 . Notons un fait intéressant: sur les 35 individus ayant un antécédent pour crime violent/sexuel entre 18 et 20 ans, 37,1\% d'entre eux ont un tel antécédent entre 27 et 29 ans. À l'inverse, sur les 275 n'ayant pas de tel antécédent au début de l'âge adulte, seulement 10,2\% affichent des antécédents pour un crime violent/sexuel entre 27 et 29 ans, attestant ainsi d'une certaine continuité à travers le temps. Pour les fins des analyses incluses ici, il a été décidé de combiner les crimes violents avec les crimes sexuels afin d'analyser les trajectoires de comportements criminels violents/sexuels. Deux facteurs appuient cette décision : 1) la diversité du comportement criminel des agresseurs sexuels (Lussier et al., 2005); 2) la présence relativement rare d'antécédents de crimes violents et sexuels dans l'histoire criminelle de ces délinquants (Hall, 1988; Lussier, 2005)².

2. Un autre point mérite notre attention ici. Notre stratégie analytique est également en lien avec les outils de prédiction actuariels. Effectivement, les outils actuariels élaborés pour prédire les risques de récidive sexuelle incluent des prédicteurs faisant référence tant aux antécédents judiciaires pour un crime violent que pour un crime sexuel (par exemple le Static-99). La distinction entre ces deux types de crime est perdue dans la comptabilisation du score actuariel et la détermination du risque de récidive. 
Délinquance résiduelle. Les analyses ont été réalisées en contrôlant pour la criminalité violente/sexuelle résiduelle des individus inclus dans cette étude. La criminalité résiduelle fait référence à toutes les charges pour un crime violent et/ou sexuel survenu après l'âge de 29 ans et précédant les charges ayant mené à l'incarcération (figure 1). Les individus inclus dans l'échantillon avaient en moyenne 45 ans à leur sortie de prison, soit entre 30 et 77 ans. Ainsi, on peut conclure qu'une période moyenne de 15 ans existe entre la fin de la période d'observation ayant servi à l'analyse des trajectoires criminelles et la période marquant la sortie de prison. Durant cette période, certains délinquants ont commis des crimes violents/sexuels pour lesquels ils ont fait l'objet de charges. En moyenne, la délinquance résiduelle observée est de 0,77 (écart-type $2,27)$. En fait, $76,5 \%$ de l'échantillon ne présente pas de délinquance résiduelle.

F I G URE 1

Schéma analytique de l'étude

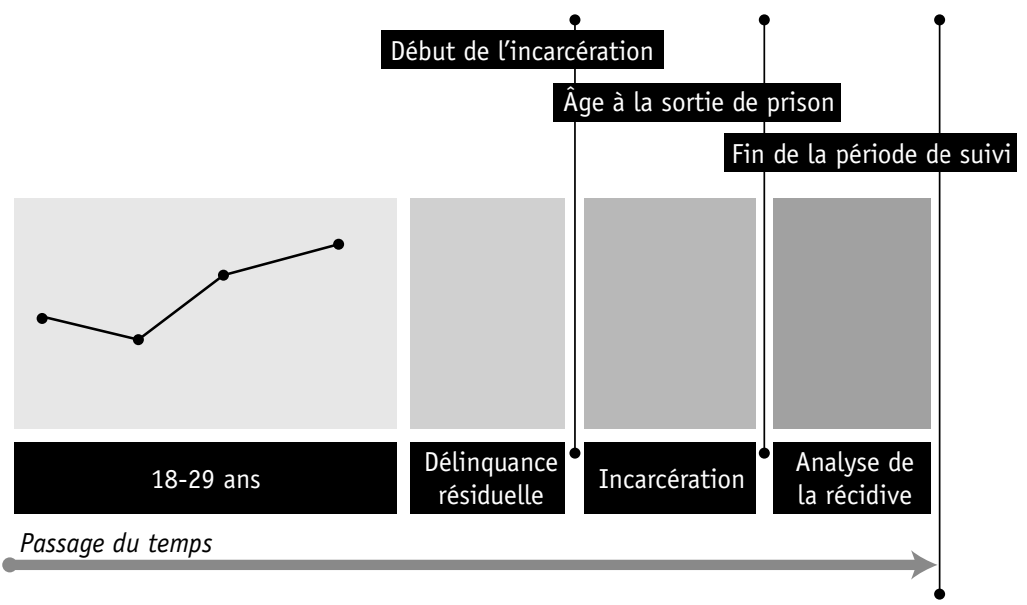

Période de suivi. La période de suivi fait référence à la durée de la période à risque de récidive pour chacun des délinquants sexuels inclus dans cette étude (figure 1). Premièrement, la date marquant la libération de prison a été notée pour chacun des délinquants inclus dans cette étude. Deuxièmement, au mois de juin 2004, les données sur la récidive ont été colligées pour chacun des sujets, marquant ainsi la fin de la période de suivi. Sur les 316 sujets inclus dans cette étude, 306 avaient été 
libérés de prison avant juin 2004. Par conséquent, 10 (3,2\%) n'avaient toujours pas été libérés et ont été exclus de l'étude. La durée moyenne de la période de suivi était de 61,5 mois (écart-type $=20,9$ ), ou environ cinq ans. La durée de la période de suivi est influencée par a) la date d'admission au pénitencier; b) la durée de la peine; c) la durée de la peine purgée; d) une récidive avant la fin de la période d’observation.

Récidive. La récidive fait référence à la présence d'une nouvelle condamnation durant la période de suivi. Une seule mesure de la récidive est utilisée pour les fins de cette étude, soit la récidive violente et/ou sexuelle. Cette récidive inclut toutes les condamnations pour un crime violent ou un crime sexuel suivant la sortie de prison. La décision de combiner la récidive violente avec la récidive sexuelle s'explique par le faible taux de récidive sexuelle $(5,2 \%)$ pour cet échantillon. Ce taux est trop faible pour permettre des analyses statistiques multivariées. Par ailleurs, une définition plus inclusive de la récidive sexuelle qui inclut les crimes violents a été utilisée maintes fois comme stratégie analytique considérant que certains individus ayant reçu des charges pour un crime sexuel ne sont condamnés que pour un crime violent grâce aux négociations entre l'avocat de la défense et celui de la Couronne. Les données descriptives sont présentées au tableau 1.

\section{Stratégie analytique}

Modèle de classes latentes. Le schéma analytique de l'étude est présenté à la figure 1. Dans un premier temps, les trajectoires criminelles d'entre 18 et 29 ans ont été analysées à l'aide de modèles de classes latentes. L'analyse de classes latentes est une méthode statistique utilisée pour identifier des classes mutuellement exclusives d'individus en fonction de leurs réponses à une série d'items (Goodman, 1974). Dans le cas qui nous intéresse, les items font référence aux variables qui mesurent le comportement criminel et la réponse à un item indique la présence ou non d'un comportement criminel à une période donnée. Ce type d'analyse permet de modéliser et d'estimer deux types de paramètres: a) $\Gamma$ ou Gamma qui représente les probabilités d'appartenance à une classe; b) $\mathrm{P}$ ou Rho qui fait référence aux probabilités de réponse de l'item conditionnelle à l'appartenance à une classe. Ainsi, les modèles de classes latentes permettent de prédire les probabilités d'appartenance des individus à chacune des classes mutuellement exclusives et exhaustives identifiées sur la base des réponses observées à chacun des items 
analysés (Dayton, 2008). Dans la présente étude, les classes latentes servent donc à retracer les trajectoires criminelles. Quatre items ont été analysés, soit la présence ou non d'activité criminelle violente/sexuelle pour chacune des périodes suivantes: a) 18-20 ans; b) 21-23 ans; c) 24-26 ans; d) 27-29 ans. La corrélation entre les items a été analysée a priori et n'a pas permis de révéler la présence d'informations redondantes (l'étendue des corrélations $=0,09$ à 0,25$)$. Considérant les études antérieures auprès d'échantillons de délinquants (Le Blanc, 2005; Piquero, 2008), différents modèles de classes latentes ont été analysés, soit des solutions variant entre deux et six classes latentes (ou trajectoires). La solution optimale a été déterminée à l'aide de la statistique G2, du paramètre Akaike (AIC), ainsi que de l'information bayésienne (BIC) tout en considérant la taille de la classe identifiée, les probabilités moyennes d'appartenance à la classe, ainsi que son interprétation. Ces analyses ont été réalisées à l'aide de SAS 9.2 (Lanza et al., 2007).

Analyses de survie. La relation entre les trajectoires criminelles et la récidive violente/sexuelle a été analysée à l'aide d'analyses de survie. Dans le contexte de la présente étude, la survie fait référence au temps (en mois) s'étant écoulé depuis la libération de l'individu sans qu'il ait commis une nouvelle infraction. Les études antérieures sur la récidive ont utilisé autant la régression logistique que la régression de Cox. La régression de Cox est une technique qui permet d'examiner si la survie (ou plutôt la non-récidive) est influencée par certains facteurs. Ce type de régression contrôle pour les données tronquées, ce qui ne peut être accompli à l'aide de la régression logistique. Dans la présente étude, les données tronquées sont importantes car elles impliquent la possibilité que les non-récidivistes récidivent advenant une plus longue période de suivi. La période de suivi s'est terminée en juin 2004 et il est fort probable que certains délinquants aient récidivé après cette période. L'analyse de survie de type Cox prévaut donc ici afin de minimiser l'impact des données tronquées sur les paramètres estimés. Ainsi, une série d'analyses de type Cox a été réalisée à l'aide du logiciel PASW 18.0 afin de déterminer la relation entre les antécédents judiciaires et la récidive, et ce, tout en contrôlant pour la durée du suivi, l'âge au moment de la sortie de prison, ainsi que la délinquance résiduelle ${ }^{3}$.

3. Avant d'effectuer ces analyses, la proportionnalité des risques de survie ainsi que la linéarité des prédicteurs ont été examinées. Tout d'abord, la proportionnalité des risques a été examinée afin de s'assurer que l'effet des prédicteurs est constant au fil du temps ou 


\section{Résultats}

\section{Identification des trajectoires}

Dans un premier temps, les trajectoires ont été examinées à l'aide de modèles de classes latentes (tableau 1). De façon générale, le BIC est l'indicateur le plus utilisé pour déterminer le nombre de classes. Toutefois, le BIC n'est pas toujours utile lorsqu'une solution complète et parcimonieuse est désirée (Bushway et al., 2003). Aussi, l'interprétation des classes doit être prise en compte afin de déterminer la solution optimale (Blokland et al., 2005). Les résultats des analyses du comportement criminel violent/sexuel montrent qu'une solution à deux ou trois catégories est optimale. Le BIC observé pour une solution à deux classes latentes est légèrement inférieur à celle à trois classes. Toutefois, la solution à trois classes a été retenue pour les fins de l'étude. Cette solution a été retenue considérant qu'elle contient trois trajectoires criminelles déjà documentées dans les écrits scientifiques. Les solutions à quatre, cinq et six classes latentes ont été rejetées considérant les résultats obtenus aux différents paramètres $\left(\mathrm{G}^{2}, \mathrm{BIC}, \mathrm{AIC}\right)$ qui n'indiquent pas d'amélioration significative du modèle. La distribution des délinquants à travers les classes ainsi que les probabilités d'appartenance pour la solution à trois classes sont présentées dans le tableau 3. Ainsi, la classe I inclut 250 sujets, comparativement à 44 pour la classe II et 17 pour la classe III. Bref, il y a une surreprésentation de délinquants au sein de la première classe identifiée. À noter, les probabilités d'appartenance moyenne sont supérieures à 0,89 pour les trois classes. En revanche, les probabilités d'appartenance hors classe (par exemple, les probabilités qu'un individu classé dans le groupe 1 appartienne au groupe 2) sont toutes inférieures à 0,06 . Ces deux résultats témoignent de la stabilité des classes identifiées. 
TA B LEA U 2

Résultats sommaires des analyses de classes latentes

\begin{tabular}{|l|c|c|c|c|}
\hline Solution & $\mathbf{G}^{\mathbf{2}}$ & AIC & BIC & BIC ajusté \\
\hline Deux classes & 17,98 & 35,98 & 69,64 & 41,09 \\
\hline Trois classes & 11,01 & 39,01 & 91,36 & 46,96 \\
\hline Quatre classes & 6,60 & 44,60 & 115,66 & 55,40 \\
\hline Cinq classes & 0,86 & 48,86 & 138,62 & 62,50 \\
\hline Six classes & 0,00 & 58,00 & 166,45 & 74,48 \\
\hline
\end{tabular}

TAB LEA U 3

Probabilités d'appartenance aux classes latentes (trajectoires)

\begin{tabular}{|l|c|c|c|c|}
\hline $\begin{array}{l}\text { Classes latentes } \\
\text { (trajectoires) }\end{array}$ & Classe I & Classe II & Classe III & $\begin{array}{c}\text { Étendue des } \\
\text { probabilités } \\
\text { d'appartenance }\end{array}$ \\
\hline Classe I $(n=250)$ & $\underline{0,98(0,09)}$ & $0,00(0,00)$ & $0,02(0,09)$ & $0,53-1,00$ \\
\hline Classe II $(n=44)$ & $0,00(0,00)$ & $\underline{0,95(0,10)}$ & $0,05(0,10)$ & $0,54-1,00$ \\
\hline Classe III $(n=17)$ & $0,06(0,05)$ & $0,04(0,10)$ & $\underline{0,89(0,08)}$ & $0,75-0,99$ \\
\hline
\end{tabular}

Les classes latentes sont présentées dans la figure 2. On constate que la classe I représente une trajectoire où les probabilités de commettre un délit violent/sexuel sont sous la barre de 0,10 entre 18 et 29 ans. Cette trajectoire présente des probabilités constantes et linéaires qui suggèrent que les risques de passage à l'acte pour ce groupe sont faibles. En comparaison, les probabilités de commettre un crime violent/sexuel de la classe II entre 18 et 29 ans sont non linéaires. Les probabilités de l'agir violent/sexuel pour cette trajectoire, qualifiée de "sporadique», avoisinent les 0,20 , sauf pour la période de 21 à 23 ans où tous les individus caractérisés par cette trajectoire ont commis un délit. L'activité criminelle est qualifiée ici de «sporadique» puisqu'elle est concentrée sur une seule période. Enfin, la classe III inclut un groupe d'individus plus criminellement actifs entre 18 et 29 ans. Ces délinquants sont criminellement actifs dès le passage à l'âge adulte (18-20), période suivie d'une légère accalmie, pour ensuite reprendre vigueur entre 24 et 29 ans, où les probabilités d'être actif avoisinent les 0,50 . Considérant ce patron d'activité criminelle, on peut le qualifier de trajectoire à «taux élevé-décroissant». 
FIGURE 2

Représentation graphique des trajectoires criminelles violentes/sexuelles

Trajectoires de comportements violents/sexuels

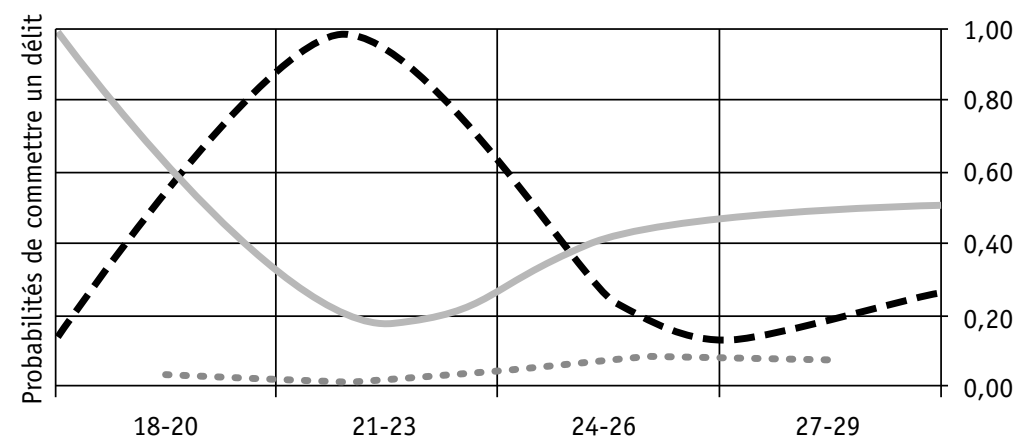

Âge du délinquant au moment de l'arrestation

-.. Classe I: faible taux

- Classe II: sporadique

Classe II: taux élevé-décroissant

Dans le tableau 4, des informations descriptives sont présentées. On constate qu'il y a des différences significatives quant à l'âge au début de l'incarcération et à la sortie de prison. Les résultats montrent que les délinquants caractérisés par une trajectoire à «faible taux» sont plus âgés à l'entrée ainsi qu'à la sortie de prison comparativement aux autres délinquants sexuels. On remarque également un effet marginalement significatif quant à la durée de la sentence (à ne pas confondre avec le temps purgé). Les délinquants caractérisés par une trajectoire «sporadique» montrent une tendance à recevoir des sentences plus longues que les autres délinquants sexuels. Notons que les analyses de variance ne montrent pas de différences statistiquement significatives entre les trajectoires criminelles quant au nombre de victimes âgées de moins de 13 ans ainsi que le nombre de victimes âgées de plus de 17 ans. Cela suggère qu'il n'y a pas de différences significatives entre les trajectoires quant au type de délinquants sexuels. Si les trajectoires criminelles ont été identifiées à partir de la participation criminelle pour quatre périodes distinctes, le tableau 4 présente le nombre de charges pour chacune de ces périodes afin de déterminer si les trajectoires se distinguent également en fonction de l'intensité de l'activité criminelle violente/ sexuelle. Les résultats des analyses de variance sont clairs à ce sujet. 
TABLEAU 4

Description des trajectoires de crimes violents et sexuels

\begin{tabular}{|c|c|c|c|c|c|}
\hline Indicateurs & Classe I: Faible taux & Classe II : Sporadique & $\begin{array}{l}\text { Classe III : Taux } \\
\text { élevé-décroissant }\end{array}$ & Test $\mathbf{F}$ & $\begin{array}{c}\text { Test } \\
\text { post-hoc }\end{array}$ \\
\hline Âge au début de l'incarcération & $43,8(11,0)$ & $36,4(7,1)$ & $35,1(5,2)$ & $13,7^{* * *}$ & I>II, III \\
\hline Durée de la sentence (année) & $4,0(2,6)$ & $5,1(4,4)$ & $3,9(1,7)$ & $2,8+$ & - \\
\hline Nombre de victimes âgées de moins de 13 ans & $0,96(1,25)$ & $0,84(1,46)$ & $0,81(2,56)$ & 0,24 & - \\
\hline Nombre de victimes âgées de plus de 17 ans & $0,36(0,74)$ & $0,65(1,40)$ & $0,38(0,50)$ & 2,15 & - \\
\hline Âge au moment de la libération & $46,4(10,8)$ & $39,43(6,9)$ & $37,8(5,7)$ & $13,6^{* * *}$ & I $>$ II, III \\
\hline \multicolumn{6}{|l|}{ Nombre de charges pour un crime violent } \\
\hline $\begin{array}{l}18-20 \\
21-23\end{array}$ & $\begin{array}{l}0,05(0,33) \\
0,00(0,06)\end{array}$ & $\begin{array}{l}0,55(2,05) \\
1,57(1,37)\end{array}$ & $\begin{array}{l}1,01(1,03) \\
0,59(1,37)\end{array}$ & $\begin{array}{r}15,9^{* * *} \\
128,7^{* * *}\end{array}$ & $\begin{array}{l}\text { III, II }>\text { I } \\
\text { II }>\text { III }>\text { I }\end{array}$ \\
\hline $24-26$ & $0,09(0,53)$ & $0,23(0,60)$ & $1,47(1,59)$ & $37,4^{* * *}$ & $\mathrm{III}>\mathrm{II}, \mathrm{I}$ \\
\hline $27-29$ & $0,07(0,34)$ & $0,23(0,64)$ & $0,59(0,62)$ & $14,6 * * *$ & III $>$ II, I \\
\hline \multicolumn{6}{|l|}{ Nombre de charges pour un crime sexuel } \\
\hline $18-20$ & $0,02(0,15)$ & $0,05(0,21)$ & $0,35(0,49)$ & $23,8 * * *$ & III $>$ II, I \\
\hline $21-23$ & $0,00(0,00)$ & $0,48(0,88)$ & $0,00(0,00)$ & $40,2^{* * *}$ & $\mathrm{II}>\mathrm{III}, \mathrm{I}$ \\
\hline $24-26$ & $0,08(0,52)$ & $0,02(0,15)$ & $0,12(0,33)$ & 0,35 & - \\
\hline $27-29$ & $0,10(0,72)$ & $0,30(0,67)$ & $0,24(0,56)$ & 0,64 & - \\
\hline \multicolumn{6}{|l|}{ Nombre de charges pour un crime violent/sexuel } \\
\hline $18-20$ & $0,07(0,37)$ & $0,59(2,08)$ & $1,35(0,79)$ & $22,38 * * *$ & $\mathrm{III}>\mathrm{II}>\mathrm{I}$ \\
\hline $21-23$ & $0,00(0,00)$ & $2,05(1,41)$ & $0,59(1,37)$ & $209,47 * * *$ & $\mathrm{II}>\mathrm{III}>\mathrm{I}$ \\
\hline $24-26$ & $0,18(0,91)$ & $0,25(0,61)$ & $1,59(1,62)$ & $18,48^{* * *}$ & III >II,I \\
\hline $27-29$ & $0,16(0,81)$ & $0,52(1,05)$ & $0,82(0,64)$ & $7,65^{* *}$ & III, II $>$ I \\
\hline Criminalité résiduelle violente/sexuelle & $0,88(2,48)$ & $0,30(0,70)$ & $0,35(1,06)$ & 1,55 & - \\
\hline
\end{tabular}

Note. Tous les tests post-hoc sont statistiquement significatifs à $p<.01$, à l'exception de la différence entre la classe I et la classe II quant à la variable du comportement criminel violent/sexuel entre 27 et 29 ans. Des analyses non paramétriques complémentaires ont été réalisées dans les situations de violation de l'homogénéité de variance. Ces résultats ne sont pas rapportés puisqu'ils vont dans le sens de ceux présentés ici. $+p<.10{ }^{*} p<.05{ }^{* *} p<.01 * * * p<.001$ 
Pour les périodes $18-20,24-26$, et 27-29 ans, la trajectoire à «taux élevé-décroissant» présente un nombre de charges significativement plus élevé que les deux autres trajectoires. Toutefois, entre 21 et 23 ans, la trajectoire «sporadique» est celle qui présente le plus grand nombre de charges pour un crime violent/sexuel. Lorsque l'on distingue les charges pour un crime violent et celles pour un crime sexuel, on remarque que les trois trajectoires criminelles présentent davantage de différences pour l'activité criminelle violente que pour la criminalité sexuelle.

Le tableau 5 présente la récidive pour les trois trajectoires des délinquants sexuels. En ce qui a trait à la récidive violente/sexuelle, on remarque des différences statistiquement significatives entre les trois trajectoires. En effet, environ $11 \%$ des individus caractérisés par une trajectoire à «faible taux» ont récidivé durant la période de suivi comparativement à $23 \%$ et $24 \%$ des individus classés respectivement dans la trajectoire «sporadique» et la trajectoire à «taux élevé-décroissant». On constate toutefois que 27 des 41 récidivistes identifiés $(65,9 \%)$ sont caractérisés par une trajectoire à «faible taux» entre 18 et 29 ans. Fait intéressant, on remarque que lorsque la récidive sexuelle est analysée séparément, il n'y a pas de différences significatives, les trois groupes présentant une proportion de récidivistes d'environ 5-6\%. Cela dit, 12 des 15 récidivistes sexuels $(80 \%)$ inclus dans cette étude sont caractérisés par une trajectoire criminelle à «faible taux» entre 18 et 29 ans. Notons que ces résultats ne tiennent pas compte de la durée de la période de suivi après la libération.

TABLEA U 5

Trajectoires criminelles et proportion de récidivistes

\begin{tabular}{|l|c|c|}
\hline Trajectoires & $\begin{array}{c}\text { Récidive violente/ } \\
\text { sexuelle }\end{array}$ & Récidive sexuelle \\
\hline Classe I: faible taux & $10,8(27)$ & $4,8(12)$ \\
\hline Classe II: sporadique & $22,7(10)$ & $4,5(2)$ \\
\hline Classe III: taux élevé-décroissant & $23,5(4)$ & $5,9(1)$ \\
\hline
\end{tabular}

Note. On constate une différence significative $\left(p^{\prime} .05\right)$ entre les pourcentages attendus et observés pour la récidive violente/sexuelle, mais pas pour la récidive sexuelle.

Les analyses de survie (régression de type Cox) permettent d'analyser le lien entre les trajectoires criminelles et la récidive violente/sexuelle en tenant compte de la durée du suivi. Les résultats sont présentés dans le tableau 6. 
TAB LEA U 6

Trajectoires criminelles et prédiction de la récidive violente/sexuelle à l'aide de modèles de survie de type Cox

\begin{tabular}{|c|c|c|c|c|c|c|}
\hline & \multicolumn{2}{|c|}{ Modèle 1} & \multicolumn{2}{|c|}{ Modèle 2} & \multicolumn{2}{|c|}{ Modèle 3} \\
\hline Indicateurs & $\begin{array}{c}\text { Rapport } \\
\text { de } \\
\text { cotes }\end{array}$ & $\begin{array}{c}\text { Intervalle } \\
\text { de confiance } \\
(95 \%)\end{array}$ & $\begin{array}{c}\text { Rapport } \\
\text { de } \\
\text { cotes }\end{array}$ & $\begin{array}{c}\text { Intervalle } \\
\text { de confiance } \\
(95 \%)\end{array}$ & $\begin{array}{c}\text { Rapport } \\
\text { de } \\
\text { cotes }\end{array}$ & \begin{tabular}{|c} 
Intervalle \\
de confiance \\
$95 \%)$
\end{tabular} \\
\hline $\begin{array}{l}\text { Âge } \\
\text { au moment } \\
\text { de la } \\
\text { libération }\end{array}$ & $0,97^{*}$ & $0,93-1,00$ & - & - & $0,97+$ & $0,94-1,00$ \\
\hline \multicolumn{7}{|l|}{ Trajectoires } \\
\hline $\begin{array}{l}\text { Classe II: } \\
\text { sporadique }\end{array}$ & - & - & 1,28 & $0,60-2,73$ & 1,25 & $0,59-2,65$ \\
\hline $\begin{array}{l}\text { Classe III: } \\
\text { élevé- } \\
\text { décroissant }\end{array}$ & - & - & $4,45^{* *}$ & $1,53-12,98$ & $3,97^{*}$ & $1,35-11,66$ \\
\hline $\begin{array}{l}\text { Modèle } \\
-2 \mathrm{LL} \\
\mathrm{G}^{2} \text { (d.l.) } \\
p\end{array}$ & \multicolumn{2}{|c|}{$\begin{array}{c}367,91 \\
4,44(1) \\
0,035\end{array}$} & \multicolumn{2}{|c|}{$\begin{array}{c}356,49 \\
8,89(2) \\
0,012\end{array}$} & \multicolumn{2}{|c|}{$\begin{array}{c}352,84 \\
12,16(3) \\
0,007\end{array}$} \\
\hline
\end{tabular}

Trois modèles ont été analysés afin de permettre une analyse de l'apport distinct de l'âge du délinquant au moment de sa libération (modèle 1), des trajectoires criminelles entre 18 et 29 ans (modèle 2), ainsi que du rôle du facteur vieillissement sur l'efficacité des trajectoires criminelles à prédire la récidive (modèle 3). Le modèle 1 montre clairement que l'âge est un prédicteur significatif de la récidive. Les résultats suggèrent que les risques de récidive violente/sexuelle diminuent de $3 \%$ par année dû au simple effet de vieillissement. Le modèle 2 présente les résultats concernant le lien entre les trajectoires criminelles et la récidive violente/sexuelle. À noter ici, afin d'examiner la relation entre les trajectoires criminelles et la récidive, qu'un groupe doit être utilisé comme référence afin de calculer les rapports de cotes. Pour ce faire, la trajectoire à «faible taux» a été sélectionnée comme groupe de référence. Les rapports de cotes qui figurent dans le tableau 6 pour les trajectoires «sporadique» et à «taux élevé-décroissant» doivent donc être interprétés à la lumière du groupe de référence. On constate que seule la trajectoire à «taux élevé-décroissant» se distingue significativement de la trajectoire de référence. Ainsi, les sujets classés dans la trajectoire à «taux élevé-décroissant» sont quatre fois plus à risque d'une récidive 
que les individus classés dans la trajectoire à «faible taux». Les courbes de survie pour les trois trajectoires criminelles sont présentées dans la figure 3. On constate que l'effet «trajectoire criminelle» débute environ deux ans $\left(20^{\mathrm{e}}\right.$ mois) après la libération pour s'accélérer après environ quatre ans et demi ( $50^{\mathrm{e}}$ mois) en communauté. On voit bien à la figure 2 que la vitesse à laquelle les délinquants sexuels caractérisés par une trajectoire «sporadique» récidivent est pratiquement identique à la vitesse à laquelle ceux caractérisés par une trajectoire à «faible taux» récidivent. Lorsque l'âge au moment de la libération et les trajectoires criminelles sont combinés, l'effet de l'âge demeure inchangé (rapport de cote $=0,97$ ), alors que celui des trajectoires criminelles diminue légèrement, le rapport de cotes passant de 4,45 à 3,97 pour la trajectoire à «taux élevé-décroissant». On remarque que l'intervalle de confiance du rapport de cotes pour les délinquants suivant une trajectoire à « taux élevé-décroissant» est relativement grand. Ceci s'explique du fait que ce groupe n'inclut que 17 individus, limitant ainsi la puissance statistique des résultats observés. En d'autres termes, ces résultats doivent être interprétés avec une certaine prudence.

Deux points méritent d'être ajoutés. Premièrement, les analyses ont également été réalisées en contrôlant pour la criminalité résiduelle qui s'est avérée non significative (les résultats ne sont pas rapportés ici, mais

F I G URE 3

Courbes de survie pour trois trajectoires criminelles

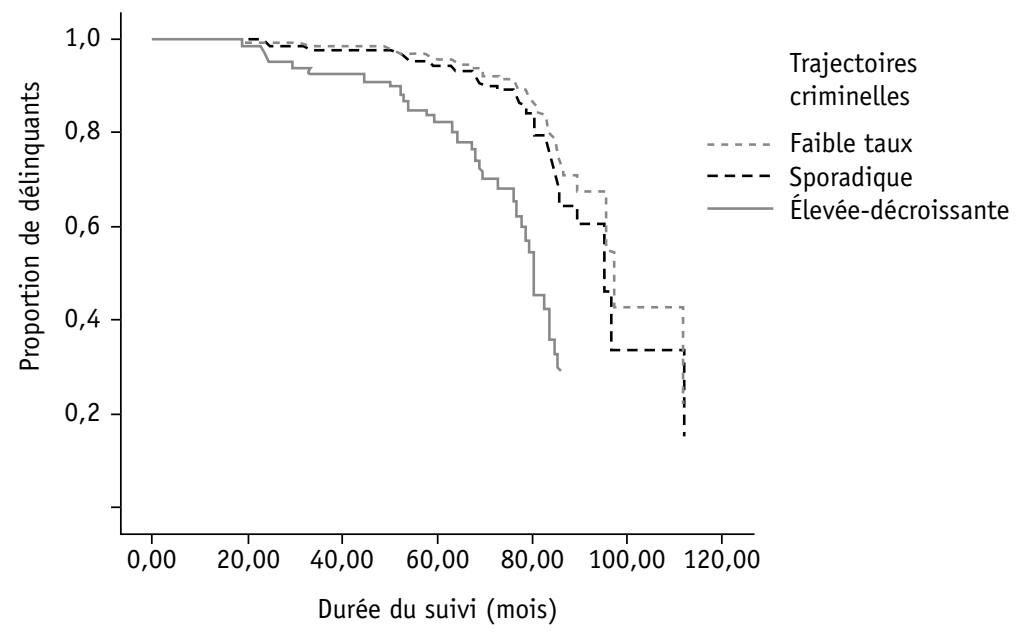


peuvent être obtenus de l'auteur). Cela est peu surprenant considérant que tous les délinquants inclus dans cette étude n'ont pas eu les mêmes opportunités de commettre des crimes violents/sexuels après l'âge de 29 ans puisqu'une proportion imposante de notre échantillon était dans la trentaine au début de l'incarcération. Deuxièmement, les analyses ont été réalisées également pour la récidive sexuelle. Aucun des prédicteurs ne s'est avéré statistiquement significatif. Cela est fort probablement attribuable au faible taux de récidive sexuelle pour les trois trajectoires criminelles analysées.

\section{Discussion}

La présente étude met en relief la complexité de la relation entre les antécédents criminels et la récidive. Il importe toutefois de commencer l'interprétation des résultats par un examen des limites méthodologiques de cette étude. L'échantillon n'inclut que des délinquants sexuels adultes incarcérés dans un pénitencier fédéral pour un crime sexuel. Les résultats ne s'appliquent donc qu'à ce sous-groupe de délinquants sexuels. L'échantillon exclut en outre les délinquants sexuels âgés de moins de 30 ans au moment de leur libération, qui constituent le groupe à plus haut risque de récidive. Les antécédents criminels, tout comme la récidive, ne reposent que sur des données officielles de la criminalité. Par conséquent, l'étude ne repose pas sur une analyse complète, mais partielle, de l'activité criminelle. Les antécédents criminels ne tiennent pas en compte des périodes de temps où les délinquants n'étaient pas à risque de commettre un délit (par exemple, période d'incarcération, hospitalisation, etc.). La période d'étude concernant l'activité criminelle antérieure a été analysée sur une période de 10 ans, ce qui peut paraître relativement court, mais se rapproche de la durée moyenne de la carrière criminelle officielle adulte (Blumstein et al., 1986; Farrington, 2005). Finalement, la date considérée pour les antécédents criminels reflète le moment où les charges ont été déposées contre l'individu. Pour l'ensemble des crimes inclus dans cette étude, une période indéterminée entre le moment où le crime a eu lieu et le moment où les charges ont été déposées n'a pas été prise en compte. L'ensemble des résultats doit par conséquent être analysé et interprété à la lumière de ces limites méthodologiques. 


\section{Perspective dynamique de l'agir criminel}

Les analyses de classes latentes ont permis d'identifier, auprès d'un échantillon de délinquants sexuels âgés d'au moins 30 ans, la présence de trois trajectoires criminelles violentes/sexuelles. Ce résultat est peu surprenant considérant que les études qui portent sur l'analyse des trajectoires criminelles violentes trouvent généralement entre trois et cinq patrons d'activités criminelles (Le Blanc, 2005 ; Piquero, 2008). Ces trois trajectoires criminelles semblent valides tant pour les agresseurs de femmes que pour les agresseurs d'enfants puisque les analyses statistiques n'ont pas permis de démontrer de lien entre le type de délinquant et l'appartenance à une trajectoire particulière (voir également Lussier et al., 2010). La première trajectoire, qui représente $80,4 \%$ de l'échantillon analysé, présente une criminalité violente/sexuelle pratiquement absente entre 18 et 29 ans. L'importance de cette trajectoire au sein de cet échantillon contraste avec l'idée selon laquelle les délinquants sexuels présentent un risque élevé de récidive. Au contraire, ce résultat va donc dans le sens d'une étude antérieure (Lussier et Davies, 2010) qui suggère que de façon générale, les délinquants sexuels âgés d'au moins 30 ans ne sont pas caractérisés par une carrière criminelle violente et sexuelle stable et persistante, mais que l'agir criminel violent et sexuel se veut davantage transitoire et discontinu. La deuxième trajectoire qui inclut les délinquants sporadiques caractérise $14,1 \%$ de l'échantillon. Ces délinquants ont tendance à commencer leur activité criminelle entre l'âge de 21 et 23 ans. En fait, durant cette période, tant leur activité criminelle violente que leur activité criminelle sexuelle sont plus élevées que celles des deux autres groupes. Lors des périodes subséquentes, toutefois, leur criminalité n'est pas différente de celle des individus inclus dans la trajectoire à «faible taux». Finalement, la troisième trajectoire, qui inclut 5,5\% de l'échantillon, caractérise une trajectoire criminelle plus précoce, plus volumineuse et plus grave que les deux autres. En effet, ces délinquants sont actifs dès le début de l'âge adulte (18-20), cette criminalité est suivie d'un ralentissement, pour ensuite reprendre une certaine vigueur. Ce type de patron criminel est probablement le résultat d'une période active d'agir criminel violent/sexuel entrecoupée de sanctions pénales (probation, incarcération, etc.). La modélisation des trajectoires criminelles en tenant compte des périodes où les délinquants font l'objet de sanctions pénales permettrait de clarifier cette hypothèse. Bref, on constate que d'une période 
à l'autre, il y a un recoupement de l'activité criminelle entre les trois groupes. Autrement dit, le groupe le plus actif dans une période n'est pas nécessairement le plus actif lors des périodes subséquentes, soulignant ainsi l'aspect dynamique de l'activité criminelle des délinquants sexuels.

\section{Âge, trajectoires criminelles et récidive}

L'importance de l'âge. Les trajectoires criminelles d'entre 18 et 29 ans ont été analysées afin de déterminer si elles comportaient une valeur prédictive significative quant à la récidive pour la période qui commence vers l'âge de 30 ans. Autrement dit, l'étude visait à examiner la présence de continuité de l'agir criminel violent/sexuel en tenant compte du facteur temps. Trois résultats importants retiennent ici notre attention, soit l'importance de l'âge au moment de la libération, la discontinuité et la continuité de l'agir criminel, et l'impact relatif des délinquants persistants. Le facteur temps a été analysé en tenant compte de l'âge du délinquant au moment de sa libération, mais également en considérant le temps passé en communauté après la libération sans commettre un nouveau crime violent/sexuel. Les résultats montrent que l'âge au moment de la libération constitue un prédicteur de la récidive violente/ sexuelle. Ce résultat est d'autant plus pertinent que le groupe le plus à risque de récidive, soit les individus âgés de moins de 30 ans au moment de la libération, a été exclu de l'étude. Bref, l'effet de l'âge observé dans les études antérieures (Barbaree et al., 2003; Thornton, 2006; Wollert, 2006; Lussier et Healey, 2009) ne peut pas être expliqué par les taux de récidive des jeunes délinquants sexuels adultes, mais suggère plutôt un effet bien connu des criminologues, soit la relation âge-crime (notamment Farrington, 2005). Plus important encore, l'effet de l'âge demeure présent (quoique marginalement significatif) lorsque les trajectoires criminelles sont prises en compte. Ainsi, ces résultats suggèrent que les risques de récidive diminuent avec l'âge, peut importe le niveau d'activité criminelle entre l'âge de 18 et 29 ans. Bref, l'effet de l'âge est présent tant chez les délinquants sporadiques que chez ceux qui présentent une trajectoire à «taux élevé-décroissant». Ces résultats vont dans le sens des résultats des travaux de Blumstein et Nakamura (2009) auprès de délinquants adultes. Effectivement, les résultats de ces travaux montrent qu'après un certain laps de temps, le risque de récidive des individus ayant commis un crime au début de l'âge adulte devient 
équivalent à celui de la population en général. Leurs travaux remettent en question l'utilité de mettre à disponibilité le casier judiciaire de ces individus avec un antécédent judiciaire lointain à de futurs employeurs, surtout lorsqu'on connait l'impact négatif de ce dévoilement sur le plan de l'intégration sociale. Il serait intéressant de vérifier l'hypothèse de Blumstein et Nakamura auprès d'un échantillon de délinquants sexuels.

La discontinuité et la continuité de l'agir criminel. L'étude des trajectoires criminelles et de leur rapport avec la récidive a permis de mettre en perspective l'importance de la continuité et de la discontinuité de l'agir criminel. L'idée n'est pas nouvelle (voir Kruttschnitt et al., 2000), mais elle a été très peu abordée dans la littérature scientifique. Premièrement, on remarque que les délinquants qui présentent une trajectoire criminelle «sporadique» montrent une activité criminelle plus active que celle des délinquants ayant suivi une trajectoire à «faible taux». Les délinquants sporadiques ont une activité criminelle violente/sexuelle plus précoce à l'âge adulte mais également plus volumineuse, tant pour les crimes violents que pour les crimes sexuels. Or, si entre l'âge de 18 et 29 ans les délinquants sporadiques se distinguent des délinquants à faible taux, ces différences s'estompent après l'âge de 30 ans, au moins pour ce qui a trait à la récidive sexuelle/violente. Si l'analyse de la récidive montre une plus grande proportion de récidivistes chez les délinquants sporadiques que chez les délinquants qui présentent une trajectoire à «faible taux», les analyses de survie, pour leur part, ne montrent pas de différences significatives quant au temps passé en communauté sans commettre un nouveau délit. C'est donc dire que, malgré la présence de facteurs de risque statiques qui caractérisent les délinquants sporadiques (c.-à-d. présence d'antécédents de crimes violents, présence d'antécédents de crimes sexuels), les risques de passage à l'acte peuvent changer avec le temps d'un individu à l'autre. Les outils actuariels ne permettent pas d'ajuster à la baisse les risques de récidive puisque les trajectoires criminelles, tout comme l'aspect dynamique du passage à l'acte, ne sont pas pris en compte dans l'évaluation du risque. De plus, ces résultats ne vont pas dans le sens des conclusions émises par Harris et Rice (2007) qui affirment qu'une criminalité plus précoce (le terme n'étant pas fermement opérationnalisé par les auteurs) est un facteur de risque associé à la récidive. Nos résultats montrent que les délinquants sporadiques commencent plus tôt que les délinquants à «faible taux», mais cela ne se traduit pas par un risque plus élevé 
lorsqu'ils sont remis en liberté. Ce résultat est d'autant plus intéressant lorsqu'on note que les délinquants «sporadiques» et les délinquants ayant suivi une trajectoire à «taux élevé-décroissant» commencent leur criminalité violente/sexuelle à quelques années d'intervalle (18-20 ans versus 21-23), mais le potentiel de récidive est distinct entre les deux groupes. On voit ici l'avantage de l'approche centrée sur la personne qui fait ressortir deux types de trajectoires dont l'activation se fait tôt (avant 24 ans), mais dont les parcours après l'activation sont différents. Ceci n'est pas sans rappeler les études sur la carrière criminelle qui soulignent la diversité des patrons de comportements criminels (Blumstein et al., 1986; Moffitt, 1993; Cusson, 1998; Piquero et al., 2003 ; Sampson et Laub, 2005).

L'impact relatif des délinquants persistants. L'approche centrée sur la personne a permis de désagréger les risques de récidive en fonction des trajectoires criminelles. Ce faisant, il a été possible de regarder la récidive en considérant la prévalence de chacune des trajectoires. Un portrait différent des études actuarielles semble émerger. En effet, d'une part, on constate que les délinquants les plus actifs entre 18 et 29 ans présentent des risques de récidive violente/sexuelle plus élevés que les deux autres groupes. On peut penser que la trajectoire à «taux élevédécroissant» inclut un plus grand nombre de délinquants à haut risque de récidive en comparaison des délinquants inclus dans les deux autres trajectoires. Ce résultat est tout à fait en lien avec l'approche actuarielle sur laquelle les politiques pénales reposent. Ces politiques visent principalement à prévenir les crimes violents et sexuels des délinquants à haut risque de récidive. D'autre part, toutefois, les résultats indiquent que le sous-groupe d'individus qui présente une trajectoire à «taux élevé-décroissant» inclut seulement 9,8\% des récidivistes violents/ sexuels et $6,7 \%$ des récidivistes sexuels. La majorité des récidivistes présentent plutôt une trajectoire à «faible taux», et dont l'activité criminelle violente et sexuelle débute plus tardivement. Si ce résultat peut paraître contradictoire, il n'en est rien et il est simplement attribuable à la faible prévalence de délinquants sexuels présentant une trajectoire criminelle à «taux élevé-décroissant», soit la plus précoce, volumineuse et persistante des trois trajectoires observées. On voit mal comment une stratégie de prévention axée uniquement sur l'identification et la neutralisation de ce petit groupe de délinquants pourrait avoir un impact significatif sur la criminalité violente/sexuelle dans la société. Bref, ces 
résultats font ressortir les limites d'une stratégie pénale axée uniquement sur l'identification et la prévention du comportement criminel violent/ sexuel d'un petit groupe de délinquants sexuels, qui n'est pas sans rappeler la stratégie pénale dont l'accent est mis sur l'identification de délinquants à haut risque de récidive.

\section{Conclusion}

Les résultats de cette étude basée sur une approche centrée sur la personne montrent la continuité et la discontinuité du comportement criminel violent et sexuel à travers le temps. Les analyses de classes latentes ont permis d'identifier la présence de trois trajectoires criminelles au sein d'un échantillon de délinquants âgés d'au moins 30 ans à leur sortie de prison. L'analyse des trajectoires montre que chez ce sous-groupe, le patron d'activité criminel violent et sexuel plus important est caractérisé par des faibles probabilités de passage à l'acte, tant sur le plan des antécédents judiciaires que de la récidive après la sortie de prison. Ce résultat met en doute la présence d'une «carrière criminelle violente et sexuelle persistante» au sein de ce groupe de délinquants, suggérant plutôt que le comportement criminel violent/sexuel est principalement transitoire et discontinu. Notons toutefois que ces conclusions reposent sur l'analyse de la criminalité officielle et une analyse de la criminalité auto-rapportée est de mise avant de tirer des conclusions fermes. L'étude met également en lumière la présence d'un petit groupe de délinquants sexuels dont la criminalité violente/sexuelle est davantage persistante à travers le temps. Les résultats montrent toutefois que ce groupe est également sujet à l'effet de l'âge et que les risques de récidive diminuent à travers le temps. De plus, ce groupe représente seulement une très faible proportion des récidivistes identifiés lors du suivi. Les résultats montrent ainsi la complexité de l'aspect dynamique de l'activité criminelle à travers le temps chez les délinquants sexuels. L'approche centrée sur la personne ne constitue pas une solution au problème de la délinquance sexuelle et de la compréhension de la récidive. Elle pose néanmoins un regard nouveau qui fait contraste avec les études actuarielles centrées sur les «variables». D’autres études devraient être réalisées afin d'évaluer l'impact du traitement et des sanctions pénales à travers les trajectoires criminelles et d'améliorer la prévention de la criminalité violente et sexuelle des délinquants sexuels adultes. 


\section{Références}

Barbaree, H. E., Blanchard, R., \& Langton, C. M. (2003). The development of sexual aggression through the life span - the effect of age on sexual arousal and recidivism among sex offenders. Annals of the New York Academy of Sciences, 989, 59-71.

Barbaree, H. E., \& Blanchard, R. (2008). Sexual deviance over the lifespan: Reductions in deviant sexual behavior in the aging sex offender. In D. R. Laws \& W. T. O’Donohue (Eds.), Sexual Deviance: Theory, Assessment, and Treatment (37-60). New York: Guilford Press.

Barbaree, H. E., Langton, C. M., \& Blanchard, R. (2007). Predicting recidivism in sex offenders using the VRAG and SORAG: The contribution of age-atrelease. International Journal of Forensic Mental Health, 6, 29-46.

Barbaree, H. E., Langton, C. M., Blanchard, R., \& Cantor, J. M. (2009). Aging Versus Stable Enduring Traits as Explanatory Constructs in Sex Offender Recidivism. Criminal Justice and Behavior, 36, 443-465.

Bergman, L. R. (2002). Studying processes: some methodological considerations. In L. Pulkkinen \& A. Caspi (Eds.), Paths to Successful Development: Personality in the Life Course (177-199). Cambridge, UK: Cambridge University Press.

Bergman, L. R., \& Magnuson, D. (1997). A person-orientated approach to research on developmental psychopathology. Development and Psychopatho$\log y, 9,291-319$.

Blumstein, A., Cohen, J., Roth, J. A., \& Visher, C. A. (1986). Criminal Careers and "Career Criminals"(Volume I). Washington, DC: National Academy Press.

Blumstein, A., \& Nakamura, K. (2009). Redemption in the presence of widespread criminal background checks. Criminology, 47, 327-359.

Cale, J, Lussier, P., \& Proulx, J. (2009). Heterogeneity in antisocial trajectories in youth of adult sexual aggressors of women: An examination of initiation, persistence, escalation, and aggravation. Sexual Abuse: A Journal of Research and Treatment, 21, 223-248.

Côté, G. (2001) Les instruments d'évaluation du risque de comportements violents: mise en perspective critique. Criminologie, 34 (1), 31-45.

Cusson, M. (1998). Criminologie actuelle. Paris: Les Presses universitaires de France.

Dayton, M. C. (2008). An introduction to latent class analysis. In S. Menard (Ed.). Handbook of Longitudinal Research: Design, Measurement, and Analysis (357-371). Burlington, MA: Elsevier Press.

Doren, D. M. (1999). Recidivism base rates, predictions of sex offender recidivism, and the "sexual predator" commitment laws. Behavioral Sciences and the Law, 16, 97-114.

Doren, D. M. (2004). Stability of the interpretative risk percentages for the RRASOR and static-99. Sexual Abuse: Journal of Research and Treatment, 16, 25-36. 
Doren, D. M. (2006). What do we know about the effect of aging on recidivism risk for sexual offenders? Sexual Abuse: Journal of Research and Treatment, 18, 137-157.

Epperson, D. L., Kaul, J. D., Huot, S. J., Hesselton, D., Alexander, W., \& Goldman, R. (1998). Minnesota sex offender screening tool - revised (MnSOST-R). St-Paul, MN : Minnesota Department of Corrections.

Farrington, D. P. (2005). Integrated life-course and developmental theories of offending. New Brunswick, NJ: Transaction Publishers.

Fazel, S., Sjöstedt, G., Långström, N., \& Grann, M. (2006). Risk factors for criminal recidivism in older sexual offenders. Sexual Abuse: Journal of Research and Treatment, 18, 159-167.

Feeley, M. M., \& Simon, J. (1992). The new penology: Notes on the emerging strategy of corrections and its implications. Criminology, 30, 449-474.

Goodman, L. A. (1974). Exploratory latent structure analysis using both identifiable and unidentifiable models. Biometrika, 61, 215-231.

Grambsch, P. M., \& Therneau, T. M. (1994). Proportional hazards tests and diagnostics based on weighted residuals. Biometrika, 81, 515-526.

Hall, G. C. N. (1988).Criminal behavior as a function of clinical and actuarial variables in a sexual offender population. Journal of Consulting and Clinical Psychology, 56, 773-775.

Hanson, R. K. (1997). The development of a brief actuarial scale for sexual offense recidivism. Ottawa, ON : Solicitor General of Canada.

Hanson, R. K. (2002). Recidivism and age: Follow-up data from 4, 673 sexual offenders. Journal of Interpersonal Violence, 17, 1046-1062.

Hanson, R. K. (2006). Does static-99 predict recidivism among older sexual offenders? Sexual Abuse: A Journal of Research and Treatment, 18, 343-355.

Hanson, R. K., \& Bussière, M. T. (1998). Predicting relapse: A meta-analysis of sexual offender recidivism studies. Journal of Consulting and Clinical Psychology, 66, 348-362.

Hanson, R. K., Gordon, A., Harris, A. J. R., Marques, J. K., Murphy, W., Quinsey, V. L., \& Seto, M. C. (2002). First report to the collaborative outcome data project on the effectiveness of psychological treatment for sex offenders. Sexual Abuse: A Journal of Research and Treatment, 14, 169-194.

Hanson, R. K., \& Thornton, D. (2000). Improving risk assessments for sex offenders: A comparison of three actuarial scales. Law and Human Behavior, 24, 119-136.

Hanson, R. K., \& Harris, A. J. R. (2001). A Structured Approach to Evaluating Change Among Sexual Offenders. Sexual Abuse: A Journal of Research and Treatment, 13, 105-122.

Harris, G. T., \& Rice, M. E. (2007). Adjusting actuarial violence risk assessments based on aging or the passage of time. Criminal Justice and Behavior, 34, 297-313.

Kruttschnitt, C., Uggen, C., \& Shelton, K. (2000). Predictors of desistance among sex offenders: The interaction of formal and informal social controls. Justice Quarterly, 17, 61-88. 
La Fond, J. Q. (2005). Preventing Sexual Violence: How Society Should Cope with Sex Offenders. Washington, DC: American Psychological Association.

Lanza, S. T., Collins, L. M., Lemmon, D. R., \& Schafer, J. L. (2007). PROC LCA : A SAS Procedure for Latent Class Analysis. Structural Equation Modelling, 14, 671-694.

Le Blanc, M. (2005). An integrative personal control theory of deviant behavior: Answers to contemporary empirical and theoretical developmental criminological issues. In D. P. Farrington (Ed.), Integrated developmental and life-course theories of offending (125-163). London: Transaction.

Le Blanc, M., \& Fréchette, M. (1989). Male Offending from Latency to Adulthood. New York: Springer-Verlag.

Levenson, J. S., D’Amora, D. A., \& Hern, A. L. (2007). Megan's law and its impact on community re-entry for sex offenders. Behavioral Sciences and the Law, 25, 587-602.

Lösel, F., \& Schmucker, M. (2005). The effectiveness of treatment for sexual offenders: A comprehensive meta-analysis. Journal of Experimental Crimino$\log y, 1,117-146$.

Lussier, P. (2005). The criminal activity of sexual offenders in adulthood: Revisiting the specialization debate. Sexual Abuse: Journal of Research and Treatment, 17, 269-292.

Lussier, P., \& Cortoni, F. (2008). The development of antisocial behavior and sexual aggression: Theoretical, empirical and clinical implications. In B. Schwartz, (Ed.), The sex offender: Offender evaluation and program strategies (2/2-2/21). Kingston, NJ: Civic Resaerch Institute.

Lussier, P., Dahabieh, M., Deslauriers-Varin, N., \& Thompson, C. (2010). The community re-entry and the risk management of high-risk sex offenders: Issues, challenges and constraints. In L. Gideon \& H. E. Sung (Eds.), Rethinking Corrections: Rebabilitation, Reentry and Reintegration (219-252). Thousand Oaks, CA: Sage. Sous presse.

Lussier, P., \& Davies, G. (2010). A Person-oriented Perspective on Sexual Offenders, Offending Trajectories, and Risk of Recidivism: A New Challenge for Policymakers, Risk Assessors, and Actuarial Prediction? En évaluation.

Lussier, P., Deslauriers-Varin, N., \& Ratel, T. (2009). A descriptive profile of high-risk sex offenders in British Columbia, Canada. International Journal of Offender Therapy and Comparative Criminology, 54, 71-91.

Lussier, P., \& Healey, J. (2009). Rediscovering Quetelet, Again: The 'Aging' Offender and the Prediction of Reoffending in a Sample of Adult Sex Offenders. Justice Quarterly, 26, 827-856.

Lussier, P., Tzoumakis, S., Cale, J., \& Amirault, J. (2010). Criminal trajectories of adult sex offenders and the age effect: Examining the dynamic aspect of offending in adulthood. International Criminal Justice Review, 20, 147-168.

MacKenzie, D. L. (2006). What works in Corrections: Reducing the Criminal Activities of Offenders and Delinquents. Cambridge, UK: Cambridge University Press. 
Meehl, P. E. (1954). Clinical versus statistical prediction: A theoretical analysis and a review of the evidence. Minneapolis, $\mathrm{MN}$ : University of Minnesota Press.

Moffitt, T. E. (1993). Adolescence-limited and life-course-persistent antisocial behavior: A developmental taxonomy. Psychological Review, 4, 674-701.

Monahan, J., Steadman, H. J., Clark Robbins, P., Appelbaum, P., Banks, S., Grisso, T., Heilbrun, K., Mulvey, E. P., Roth, L., \& Silver, E. (2005). An actuarial model of violence risk assessment for persons with mental disorders. Psychiatric Services, 56, 810-815.

Nuffield, J. (1982). Parole Decision-Making in Canada: Research Towards Decision Guidelines. Ottawa, ON : Solicitor General of Canada.

Petrunik, M. (2002). Managing unacceptable risk: Sex offenders, community response, and social policy in the United Stated and Canada. International Journal of Offender Therapy and Comparative Criminology, 46, 483-511.

Petrunik, M. (2003). The hare and the tortoise: Dangerousness and sex offender policy in the United States and Canada. Canadian Journal of Criminology and Criminal Justice, 45, 43-72.

Piquero, A. R., Farrington, D. P., \& Blumstein, A. (2003). Criminal career paradigm. In M. Tonry (Ed.), Crime and Justice: A Review of Research (359506). Chicago: Chicago University Press.

Prentky, R. A., \& Lee, A. F. S. (2007). Effect of age-at-release on long term sexual re-offense rates in civilly committed sexual offenders. Sexual Abuse: Journal of Research and Treatment, 19, 43-59.

Prentky, R. A., Lee, A. F. S, Knight, R. A., \& Cerce, D. (1997). Recidivism rates among child molesters and rapists: A methodological analysis. Law and Human Behavior, 21, 635-659.

Proulx, J., Lamoureux, B., Tardif, M., \& Lussier, P. (2000). How does recidivism risk assessment predict survival? In D. R. Laws, S. M. Hudson \& T. Ward (Eds.), Remaking relapse prevention with sex offenders: A sourcebook (466-484). Thousand Oaks, CA: Sage.

Quinsey, V. L., Rice, M. E., \& Harris, G. T. (1995). Actuarial prediction of sexual recidivism. Journal of Interpersonal Violence, 10, 85-105.

Quinsey, V. L., Harris, G. T., Rice, M. E., \& Cormier, C. A. (1998). Violent Offenders: Appraising and Managing Risk. Washington, DC: American Psychological Association.

Roberts, C. F., Doren, D. M., \& Thornton, D. (2002). Dimensions associated with assessments of sex offender recidivism risk. Criminal Justice and Behavior, 29, 569-589.

Sampson, R. J., \& Laub, J. H. (2005). A life-course view of the development of crime. Annals of the American Academy of Political and Social Science, 602, 12-45.

Sarbin, T. R. (1943). A contribution to the study of actuarial and individual methods of prediction. American Journal of Sociology, 48, 593-602.

Silver, E., Smith, W. R., \& Banks, S. (2000). Constructive actuarial devices for predictive recidivism: A comparison of Methods. Criminal Justice and behavior, 27, 733-764. 
Simon, J. (1998). Managing the monstrous: Sex offenders and the new penology. Psychology, Public Policy, and Law, 4, 452-467.

Simon, L. M. J. (2000). An examination of the assumptions of specialization, mental disorder, and dangerousness in sex offenders. Behavioral Sciences and the Law, 18, 275-308.

Tabachnick, B. G., \& Fidell, L. S. (2007). Using multivariate statistics (5th ed.). Boston, MA: Allyn \& Bacon/Pearson Education.

Tewksbury, R. (2005). Collateral consequences of sex offender registration. Journal of Contemporary Criminal Justice, 21, 67-82.

Therneau, T. M., Grambsch, P. M., \& Fleming, T. R. (1990). Martingale-based residuals for survival models. Biometrika, 77, 147-160.

Thornberry, T. P., \& Krohn, M. D. (2005). Applying interactional theory to the explanation of continuity and change in antisocial behavior. In D. P. Farrington (Ed.), Integrated Developmental and Life Course Theories of Offending Advances in Criminological Theory (Vol. 14). Piscataway, NJ: Transaction.

Thornton, D. (2006). Age and sexual recidivism: A variable connection. Sexual Abuse: Journal of Research and Treatment, 18, 123-135.

Travis, J., \& Petersilia, J. (2001). Reentry reconsidered: A new look at an old question. Crime and Delinquency, 47, 291-313.

Von Eye, A., \& Bogat, G. A. (2006). Person-Oriented and Variable-Orientated Research: Concepts, Results and Development. Merrill-Palmer Quarterly, 52, 390-420.

Von Eye, A., \& L. R. Bergman. (2003). Research strategies in developmental psychopathology: Dimensional identity and the person-orientated approach. Development and Psychopathology, 15, 553-580.

Ward, T. \& Beech, A. (2006). An integrated theory of sexual offending. Aggression and Violent Behavior, 11, 44.63.

Wollert, R. (2006). Low base rates limit expert certainty when current actuarials are used to identify sexually-violent predators. Psychology, Public Policy, and Law, 12, 56-85.

ABSTRACT • Generally speaking, risk assessment tools are based on static or historical "predictors" such as criminal history. This study revisits the relationship between the criminal history and violent/sexual recidivism using a longitudinal perspective by taking into account the dynamic aspect of criminal careers. More specifically, the study attempted to answer the following question: Are offending trajectories carrying any predictive value to help informing about the risk of reoffending following prison release? The study is based on a quasi-population of adult sex offenders incarcerated in the province of Quebec between 1994 and 2000. Latent class analyses were performed to identify the presence of offending trajectories. Survival analyses showed that offending trajectories do inform about the risk of violent/sexual reoffending, but in doing so, highlighted some important limitations of current actuarial prediction methods.

KEYWORDS - Sex offenders, criminal trajectories, actuarial prediction, recidivism, risk assessment, dangerousness, age, criminal record. 
RESUMEN - De manera general, la evaluación de riesgo reposa sobre herramientas actuariales que incluyen factores estáticos o históricos tales como los antecedentes criminales. Este estudio reexamina la relación entre los antecedentes criminales y la reincidencia violenta/sexual tomando en cuenta el aspecto dinámico de la carrera criminal. El estudio intenta responder a la siguiente pregunta: ¿las trayectorias criminales informan sobre los riesgos de reincidencia? El presente estudio se basa en una muestra de delincuentes sexuales adultos encarcelados en la provincia de Quebec entre 1994 y 2000. Se realizaron análisis de clases latentes con el fin de identificar las trayectorias criminales violentas/sexuales. Los análisis de persistencia muestran que las trayectorias criminales informan sobre los riesgos de reincidencia violenta/sexual, pero muestran ciertos límites de las predicciones actuariales.

PALABRAS CLAVE - Delincuentes sexuales, trayectorias criminales, predicción actuarial, reincidencia, evaluación de riesgo, peligrosidad, edad, antecedentes judiciales. 